\title{
5.
}

\section{Immunitätsreaktionen nach der Vorimpfung mit artfremden Tumoren.}

Von

Prof. Dr. Carl Lewin.

Bekanntlich gelingt es, gegen hochvirulente Mäuse- und Rattentumoren eine künstliche Inmunisierung durch Torimpfung mit avirulentem Geschwulstmaterial herbeizufithren. Derselbe Effekt lässt sich erreichen, wenn zur Vorimpfung normale Gewebszellen - Embryonalzellen, rote Blutkörperchen, Milzgewebe usw. - verwendet werden. Diese Tatsachen sind von so vielen Seiten bestätigt worden, dass sie jetzt wohl allgemeine Gültigkeit erlangt haben. Die Immunisierung gelingt auch, wenn zur Vorbehandlung eine andere Tumorart genommen wird, etwa Carcinom gegen Sarkom und umgekehrt. Auch die durch Vorimpfung mit normalen Gewebselementen erzielte Immunität gilt im allgemeinen gegen die manniglachsten Tumorarten. Schon hier haben sich indes manchmal Abweichungen herausgestellt. Gierke spricht davon, dass neben der Panimmunität, wie sie Ehrlich bezeichnet hat, noch eine spezielle Immunitit existiert. So konnte er durch Vorimpiung mit einem avirulenten hämorrhagischen Mäusecarcinom einen betrïichtlich höheren Grad von Immunitï gegen ein verimpibares hämorrhagisches Carcinom, also gegen einen gleichartigen Tumor, erzielen, als gegen einen Tumor vom Typus der Jensenschen Geschwulst, der in seiner Struktur vom Typus der vorgeimpiten Geschwulst sich unterscheidet. Bashford und seine Mitarbeiter glauben, dass die Resistenz der immunisierten Tiere bei der nachfolgenden Impfung im wesentlichen abhängt von dem Grade, wie das vorgeimpfte Gewebe dem zur Resistenzprïlung verwendeten zweitgeimpften Tumor histologisch nahesteht. Ich habe mehrfach darauf hingewiesen, dass dieser Satz, in diesẹr Allgemeinheit ausgesprochen, nicht richtig ist. Die schon von Ehrlich festgestellte Tatsache, dass eine Vorimpfung. mit hämorrhagischem Mäusecarcinom auch gegen eine nachfolgende Sarkomimplung schiitzt, spricht schon ohne weiteres dagegen. Bashford selbst hat ja, wie ich ebenfalls, durch In- 
jektion von Blut einen sehr weitgehenden Grad von Immunität erzielen können, obwohl doch hier die histologische Verschiedenheit von vorgeimpiter und später inokulierter Gewebsart handgreiflich ist. Auch ist ja die Tatsache, dass ich mit einem Rattencarcinom und zwar sowohl mit Adenocarcinom wie mit Kankroid gegen das Jensensche Spindelzellensarkom der Ratte fast in $95 \mathrm{pCt}$. Immunität erzielen konnte, ein Beweis gegen die Anschauung von Bashford. Nach meinen Erfahrungen, die ich immer wieder bestätigt gefunden habe, sind für den verschiedenen Ausfall solcher Versuche nicht histologische Verschiedenheiten der Tumoren verantwortlich zu machen. Vielmehr liegt die Ursache, weshalb in dem einen Falle die Vorimpfung schütt, in dem anderen nicht, lediglich in biologischen Differenzen, deren Art wir nicht kennen. Ist es mir doch oft genug vorgekommen, dass selbst Spindelzellensarkome gegen Spindelzellensarkome nicht immunisieren, während sehr differente Gewebsarten einen weitgehenden Schutz gegen Nachimpfungen mit den verschiedensten Tumorarten gewährten, wie ja auch Bridré und Borrel gerade die Milz besonders geeignet für Immunisierungszwecke gefunden haben. Für die Bedeutung biologischer Differenzen spricht auch die Beobachtung von Moreschi, der nach Vorbehandlung mit laktierenden Mäusemilchdrüsen eine ausgesprochene Immunisierung gegen 2 Mäusecarcinome des Frankfurter Instituts festgestellt hat, während gegen ein anderes Carcinom keine Spur von Immunität sich zeigte. Ich habe mit denselben Rattentumoren, welche gegen das Jensensche Spindelzellensarkom weitgehendste Immunität verursachten, gegen das Flexner-Joblingsche Rattencarcinom keine Immunität erzielen können. Dass hier keine histologischen, sondern biologische Differenzen im Spiele sein müssen, liegt wohl klar auf der Hand.

Indessen sind alle diese Immunisierungseffekte erreicht worden durch die Vorimpfung mit arteigenem Gewebe. Orth hat seinerzeit hervorgehoben, dass bei allen diesen Erscheinungen als Ursache nicht eine spezifische Wirkung anzusehen sei, sondern dass wir es hier mit einer generellen Wirkung zu tun haben, welche allein artgleichen Zellen zukommt, ob das nun Blutzellen oder Carcinomzellen oder Sarkomzellen sind.

Schon in meinen ersten Arbeiten über diesen Gegenstand habe ich die Tatsache mitgeteilt, dass es mir gelungen ist, durch Vorimpfung von Rattentumoren bei Mäusen einen deutlichen Schutz gegen nachfolgende Implungen mit hochvirulenten Mïusetumoren zu erzielen. Umgekehrt konnte ich nach der Vorimpfung von Mänsetumoren bei Ratten gegen Rattentumoren, wenn auch in weniger hohem Grade, immunisieren. Das schien darauf hinzudeuten, dass in der Artgleichheit der zur Schutzimpiung und Resistenzprüfung verwendeten Gewebe nicht allein die Lrsache der zu beobachtenden Immunitätserscheinungen bei den bösartigen Tumoren liegen kann, 
sondern dass vielleicht doch noch nühere Beziehungen zwischen den Tumoren der verschiedenen Tiere bestehen können. wie ja auch Westenhoeffer die Ansicht vertritt, dass die Geschwulstzellen aller Tierarten als wesensgleich anzusehen wären, und dass prinzipielle Cnterschiede nicht bestehen.

Bashford und seine Mitarbeiter Murray und Haaland sind nun im Gegensatz zu diesen meinen Versuchen zu dem Resultat gekommen, dass die Resorption von Tumormaterial fremder Tierarten nicht nur keine Resistenz gegen weitere Impfungen mit artgleichen Tumoren hervorruft, sondern dass die Resistenz durch die Vorbehandlung sogar vermindert wird. Also nicht Immunität, sondern Ueberempfindlichkeit tritt auf, die sich hier in dem besseren Angehen der Tumoren äussert. Nun wissen wir ja heute, wie nahe Ueberempfindlichkeits- und Immunitaitserscheinungen bei einander liegen. Auch in der Serologie lassen sich beide nicht trennen und werden heute allgemein als gleiche Phänomene aufgefisst. Hält doch die Mehrzahl der Autoren (Weichardt, Friedberger, Dörr, Friedemann, WolflEisner u. a.) daran fest, dass die anaphylaktischen Antikörper mit den übrigen Eiweissantikörpern identisch sind und speziell Friedemann weist darauf hin, dass bei der zellulären Anaphylaxie eine vollkommene Identität der anaphylaktischen Ambozeptoren mit den cytolytischen anzunehmen sei.

Inzwischen hat Moreschi nun Versuche veröffentlicht, aus denen eine direkte Bestätigung meiner ersten Angaben hervorgeht. $\mathrm{Zu}$ gleicher Zeit schienen diese Versuche aber auch eine Erklärung für die abweichenden Befunde der englischen Autoren geben zu können, Moreschi fand nämlich, dass die Vorbehandlung von Mäusen mit einem Rattensarkom gegen zwei verschiedene Mäusecarcinomstämme eine deutliche Immunität hervorruft, wenn das Zeitintervall zwischen Vor- und Nachimpinng 22-28 Tage betrug. Auch die Vorbehandlung mit Rattenmilchdrüisen rief bei denselben Mäusecarcinomstämmen bei einem Zeitintervall von 30 bis 37 Tagen eine deutliche Immunität hervor. Dagegen begünstigt die Vorbehandlung mit Rattenmilchdrüsen das Wachstum derselben Miüsetumoren, wenn das Zeitintervall zwischen Vorbehandlung und Tumorimpfung ? bis 15 Tage beträgt. Die laktierende Meerschweinchenmilchdrüse besitzt eine mässige Immunisierungskraft gegen eins der vorher erwïhnten Mäusecarcinome (den Stamm 11) und keine begiinstigende Wirkung auf das Wachstum, auch wenn das Zeitintervall zwischen Vorbehandlung und Tumorimpfung nur 8 bis 12 Tage beträgt.

Aus diesen Versuchen von Moreschi geht also klar hervor, dass durch die Vorbehandlung mit artfremden Tumoren sowohl wie mit artfremdem Vilchdrüsengewebe eine deutliche Immunisiernng gegen nachfolgende Impiungen mit arteigenen Tumoren erzielt werden kann. $\mathrm{Zu}$ 
gleicher Zeit allerdings wird die Angabe von Bashford, Murray und Haal and bestätigt, die bei ihren dahingehenden Versuchen eine Wachstumsbegïnstigung gesehen haben. Das Entscheidende dabei ist nach Moreschi das Zeitintervall zwischen Vorimpfung und Tumorimpfung, Ich habe diesen Fragen schon seit längerer Zeit meine Aufmerksamkeit zugewendet und sehr viele Versuche mit Vorbehandlung von artfremden Tumoren angestellt. Zur Vorbehandlung nahm ich Menschencarcinom, Katzencarcinom bei Ratten und Mäusen, ferner Rattencarcinom und Sarkom bei Väusen, Mäusecarcinom und Sarkom bei Ratten.

\section{Versuch bei Katzencarcinom.}

Zur Vorbehandlung wurde ein Carcinom der Mamma von einer Katze verwendet, das am 20. Juli auf Katzen und Ratten verimpit wurde.

20. Juli. 20 Ratten werden teils subkutan, teils intraperitoneal mit Carcinom der Katze injiziert.

Am 24. Juli werden die vorbehandelten Ratten mit einem Rattencarcinom geimpft. Zur Kontrolle werden 25 Ratten mit demselben Tumor geimpit.

3. August. Von 20 Versuchstieren leben 16 . Davon 14 mit Tumoren $=87,5 \mathrm{pCt}$. positiv.

Von 25 Kontrollen leben 22. Davon 12 mit Tumoren $=54,5 \mathrm{pCt}$. positiv.

Es hat also die 4 Tage vorher vorgenommene Impfung der Ratten mit einem Katzencarcinom auf die nachfolgende Impfung mit Rattencarcinom einen deutlich begünstigenden Einfluss auf das Wachstum der Tumoren ausgeübt.

\section{Versuche mit Menschencarcinom.}

a.

1. Februar. Es werden 30 Mäuse mit Uteruscarcinom vom Menschen gespritzt. Injektion wiederholt am 6 . Februar.

20. Februar. Impfung der vorbehandelten Näuse mit Tumor D 2 a (Mäusecarcinom). Impfung von 30 Kontrolltieren.

5. März. Von 30 Kontrolltieren leben 20. Davon haben 12 Tumoren $=60$ pCt. positiv.

Von 30 vorbehandelten Tieren leben 15. Davon 2 Tumoren $=13,33$ pCt. positiv.

23. März. Von den Kontrolltieren leben 12. Davon 5 mit Tumoren $=41,6$ pCt. positiv.

Von den Versuchstieren leben, 7 . Davon 1 Tumor $=14, \ddot{\partial}$ pet.

Die 20 bzw. 14 Tage vorhergegangene Impfung mit einem Menschencarcinom hat also bei Wäusen eine deutliche Schutzwirkung gegen die Impfung mit einem Mäusecarcinom ausgeübt.

b.

15. Februar. Vorimpiung mit Carcinoma uteri vom Jenschen. 30 Mäuse.

21. Februar. Impfung der vorbehandelten Mäuse mit Mäusecarcinom D 3 a. Dazu 30 Kontrollimpfungen.

8. Härz. Unter 14 noch lebenden Versuchstieren 7 Tumoren $=50 \mathrm{pCt}$. positiv, Unter 18 noch lebenden Kontrolltieren 6 Tumoren $=331 / 3 \mathrm{pCt}$. 
Die 6 Tage vor der Impfung mit einem Mäusecarcinom vorangegangene Impfung mit einem Menschencarcinom hat eine deutlich wachstumsfördernde Wirkung auf die Entwicklung des Mäusetumors gehabt.

Ein anderer Versuch mit Menschencarcinom (Carc. mammae) ergab ein wenig ausgesprochenes Ergebnis. Es war eine deutliche Einwirkung auf das Wachstum der Tumoren weder durch \pm noch durch 16 Tage vorangegangene Vorimpfung mit dem Mammacarcinom des Menschen nachzuweisen. Aus geringfügigen Unterschieden liessen sich keine Folgerungen ziehen.

Aus beiden Versuchsreihen geht also hervor, dass die $4-6$ Tage vor der Impfung erfolgte Vorbehandlung mit Menschen- bzw. Katzencarcinom in manchen Fällen eine wachstumsbefördernde Wirkung auf nachfolgende Impfungen mit Ratten- bzw. Mäusecarcinom hat. Die 20 Tage vorher vorgenommene Vorbehandlung mit einem Menschencarcinom hat in einem Falle eine deutliche Immunität gegen ein Mäusecarcinom gezeitigt. Ein anderer gleichsinniger Versuch blieb negativ.

\section{Vorbehandlung von Mäusen mit Rattentumoren.}

a.

26. März. 30 Mäuse werden mit Rattentumor $R$ (Carcinom) geimpft.

16. April. Die vorbehandelten Mäuse werden mit Mäusecarcinom X 3 a geimpft. Ebenso 40 Kontrolltiere.

28. April. Von den vorbehandelten Mäusen leben 15. Davon 4 mit Tumoren $=26$ pCt. positiv.

Von den Kontrolltieren leben 31. Davon 17 mit Tumoren $=54 \mathrm{pCt}$. positiv.

Demnach zeigen die 21 Tage vorher mit Rattencarcinom vorbehandelten Mïuse bei der Nachimpfung mit Mäusecarcinom eine deutlich ausgesprochene Immunität (50 pCt. weniger Tumoren gegenüber den Kontrollen).

b.

Am 18. Mai werden 30 Mäuse mit Carcinom $R$ von der Ratte geimpft.

8. Juni. Es leben noch 21 vorbehandelte Mäuse. Impfung mit Mäusecarcinom CT.2. Ebenso werden 30 Kontrollmäuse geimpft.

22. Juni. Es leben noch 18 Versuchstiere, darunter 3 mit Tumoren $=16,6 \mathrm{pCt}$. positiv.

Von den Kontrollen leben 21. Davon 13 mit Tumoren $=61,8 \mathrm{pCt}$. positiv.

Die 20 Tage vor der Implung mit einem Mäusecarcinom erfolgte Vorbehandlung mit einem Rattencarcinom erzeugt eine weitgehende Immunisierung gegen das Müusecarcinom.

c.

Im 10. Mai werden 30 Mäuse mit Rattencarcinom $\mathrm{R}$ geimpit.

Am 20. Mai werden die Mäuse mit einem Mäusecarcinom CU 1 geimpft. Ebenso werden 30 Kontrollmäuse geimpft.

5. Juni. Von den Versuchstieren leben 13, darunter 8 mit Tumoren $=61,5 \mathrm{pCt}$. Von den Kontrolltieren leben 24 , davon 16 mit Tumoren $=66,5$ pCt. 
Es zeigt sich ein unerheblicher immunisierender Einfluss der 10 Tage vorher vorgenommenen Implung des Rattencarcinoms anf das Yäusecarcinom.

d.

15. Juli. Es werden 30 Müuse mit Rattencarcinon R 7 vorgcimpft. geimpet.

19. Juli. Impifung mit Mäusecarcinom CU1. Ebenso werden 30 Kontrolltiere

7. August. Von den vorbehandelten Mäusen leben 15. Davon 3 mit Tumoren $=20 \mathrm{pCt}$.

Von den Kontrollmäusen leben 17. Daron 7 mit Tumoren $=41,2 \mathrm{pCt}$.

Die Vorbehandlung mit einem Rattencarcinom 14 Tage vor der Impiung hatte also einen ersichtlichen Immunisierungseffekt gegen ein Mäusecarcinom.

c.

15. - Lugust. 30 Mäuse werclen mit Rattenearcinom R Sc vorbehandelt.

20. August. Impfung der rorbehandelten Mäuse nit Iäusecarcinom $A$. 30 Kiontrollimpfungen.

4. September. Von den vorceimpften Tieren leben 1s. Darunter \pm sehr kleine Tumoren $=22,2 \mathrm{pCt}$

Von den Kontrolltieren leben 29. Darunter 23 mit sehr grossen Tumoren $=79,3 \mathrm{pCt}$.

Die ¿ Tage vor der Impfung mit einem Mäusecarcinom vorgenommene Injektion eines Rattencarcinoms hatte einen erheblichen Immunisierungseffekt.

f.

8. September. 30 Mäuse werden mit Rattencarcinom geimpft.

15. September. Dieselben Mäuse werden mit Mäusecarcinom F reimpft. Dazu 25 Kontrollen.

17. September. Die mit Rattentumor vorgeimpften Tiere sind sümtlich eingegangen.

g.

17. Dezember 1910. 30 Mäuse werden mit Rattensarkom rorgeimptt.

21. Dezember. Dieselben Mïuse werden mit Mäusecarcinom J geimpft. Dazu -25 Kontrolien.

7. Januar 1911. Von den vorbehandelten Mäusen leben 21. Darunter sind 5 Tumoren $=23,8 \mathrm{pCt}$.

Von den Kontrolltieren leben 23. Darunter 11 mit Tumoren $=47,8 \mathrm{pCt}$.

Die 4 Tage vor der Implung vorgenommene Injektion eines Rattensarkoms hat gegen die nachlolgende Impfung eines Häuscearcinoms also eine erhebliche Schutzwirkmng ausgeübt.

$$
\text { h. }
$$

Im 16. Mai 1911 werden 60 Mäuse mit Rattensarkom $s$ rorgeimpft. trolltieren.

Am 26. Mai. Impfung mit Häusecarcinom E 11 a. Dazu Impfung von 30 Kon-

5. Juni. Von den vorbehandelten Tieren leben 48. Darunter 18 mit Tunoren $=37,5 \mathrm{pCt}$.

Von den Kontrolltieren leben 22. Darunter 20 mit Tumoren $=91 \mathrm{pCt}$.

Die 10 Tage vor der Imphung mit einem Mäusecarcinom vorgenommene Injektion von Rattencarcinom hatte gegen die Impfung des lläusecarcinoms auch hier eine erhebliche Immunisierung veranlasst. 
IV. Versuche mit Vorimpiung von Mäusetumoren bei Ratten.

a.

5. und 10. August. 20 Ratten werden mit Mäusecarcinom A vorgeimpft.

16. August. Die vorbehandelten Ratten werden mit Rattensarkom $S$ geimpft. Ebenso 20 Kontrolltiere.

28. August. Es leben noch 18 Versuchsratten. Darunter 15 mit Tumoren $=83,3 \mathrm{pCt}$. positiv.

Von den Kontrollen leben 16. Darunter 9 mit Tumoren $=56,2 \mathrm{pCt}$. positiv.

Eine 11 und 6 Tage vor der Impfung vorgenommene Impfung mit Mäusecarcinom hatte eine wachstumsbefördernde Wirkung auf die nachfolgende Impfung mit Rattensarkom.

b.

10. September. 20 Ratten werden mit Mäusecarcinom A vorgeimpft.

26. September. Dieselben Ratten mit Rattensarkom $S$ geimpft. Ebenso 20 Kontrolltiere.

10. Oktober. Von den vorbehandelten Ratten leben 18. Darunter sind 14 mit Tumoren $=77,7 \mathrm{pCt}$. positiv.

Unter 16 lebenden Kontrolltieren sind 6 mitTumoren $=37,5 \mathrm{pCt}$. positiv.

Die 16 Tage vor der Impfung vorgenommene Injektion eines Mäusecarcinoms hat gegen die nachfolgende Impfung mit einem Rattensarkom eine deutliche Schutzwirkung ausgeübt.

c.

15. April. 30 Ratten werden mit Mäusecarcinom A G vorgeimpft.

21. April. Dieselben Ratten werden mit Rattensarkom G geimpft. Dazu 30 Kontrolltiere.

5. Oktober. Von den vorbehandelten Tieren leben 21. Davon 18 mit Tumoren $=85,7 \mathrm{pCt}$.

Von den Kontrolltieren leben 26. Davon 18 mit Tumoren $=69,2 \mathrm{pCt}$.

Es hat demnach die 6 Tage vorangegangene Vorimpfung von Mäusecarcinom bei Rattensarkomen eine wachstumsbefördernde Wirkung gehabt.

d.

21. April. 30 Ratten werden mit Mäusecarcinom B vorgeimpft. trollen.

12. Mai. Die Ratten werden mit Rattensarkom G geimpft. Dazu 30 Kon-

25. Mai. Es leben 19 Versuchstiere. Davon 16 mit Tumoren $=84,2 \mathrm{pCt}$. Von den Kontrolltieren Jeben 25. Davon 21 mit Tumoren $=84 \mathrm{pCt}$.

Ein Einfluss der Vorimplung von Mäusecarcinom 21 Tage vor der Impfung eines Rattensarkoms hat sich nicht gezeigt.

e.

1. Oktober. 25 Ratten werden mit Mäusecarcinom $A$ geimpft.

5. Oktober. Dieselben Ratten werden mit Rattensarkom $\mathrm{T}$ geimpft. Ebenso 30 Kontrolltiere.

18. Oktober. Von den Versuchstieren leben 20. Darunter 10 mit Tumor $=50 \mathrm{pCt}$.

Von den Kontrollen sind 20 am Leben. Darunter 14 mit Tumoren $=70 \mathrm{pCt}$. 
Hier ist also durch die 4 Tage vorhergegangene Behandlung mit Mäusecarcinom eine nicht erhebliche Immunität gegen die nachfolgende Impfung mit Rattensarkom entstanden.

$$
\text { f. }
$$

10. Oktober. 30 Ratten geimpft mit Mäusecarcinom E. trolltiere.

25. Oktober. Dieselben Ratten mit Rattensarkom $T$ geimpft. Dazu 30 Kon-

15. November. Von den Versuchstieren sind 16 am Leben. Davon 6 mit Tumoren $=37,5 \mathrm{pCt}$.

Von den Kontrollen leben 26. Davon haben 11 Tumoren $=42,3 \mathrm{pCt}$.

Die 15 Tage vor der Impfung vorgenommene Injektion von Mäusecarcinom hat einen nennenswerten Einfluss auf die Tumorentwicklung nach Impfung mit Rattensarkom nicht gehabt.

Es geht also aus diesen Versuchen insgesamt herror. dass eine sichtliche Beeinflussung des Tumorwachstums durch die Vorimpiung mit artfremden Tumoren in sehr vielen Fällen stattfindet. Die Vorbehandlung mit einem Carcinom der Katze 4 Tage vor der Impfung hat in einem Falle auf eine nachfolgende Impfung mit Rattencarcinom einen zweifellos wachstumsbefördernden Einfluss ausgeübt $(87,5 \mathrm{pCt}$. Angangsziffer gegen 54,5 pCt. der Kontrollen). Die Vorbehandlung mit Menschencarcinom hat in einem Falle bei einem Mäusecarcinom eine wachstumsbefördernde Wirkung gehabt, wenn zwischen Vorbehandlung und Impfung ein Intervall von 6 Tagen lag. Bei einem Zeitintervall von 14 Tagen war dagegen eine Immunität gegen die nachfolgende Impfung mit Mäusecarcinom zu erzielen. Ein anderer gleichartig angelegter Versuch hatte kein ausgesprochenes Ergebnis. Es waren nennenswerte Unterschiede in der Angangsziffer der Tumoren nicht zu beobachten.

Bei der Impfung von Mäusen mit Rattenearcinom und Sarkom konnte ich in fast allen Fällen eine mehr oder minder ausgesprochene Immunisierung gegen nachfolgende Impiungen mit Mäusecarcinom erzielen. Einen Einfluss des Zeitintervalls habe ich dabei nicht konstatieren können.

Die Vorbehandlung von Ratten mit Mäusecarcinom ergab in einem Falle eine wachstumsbefördernde Wirkung bei einem Zeitintervall von 6 Tagen zwischen Vorimpfung und Impfung mit Rattensarkom. Bei Verwendung eines Zeitintervalls von 16 Tagen war in demselben Falle eine deutliche Immunität durch die Vorbehandlung nachzuweisen. In einem anderen Falle konnte eine wachstumsbefördernde Wirkung bei einem Zeitintervall von 6 Tagen $z$ wischen Vorbehandlung und Impiung ebenfalls konstatiert werden. Eine Schutzwirkung konnte ich bei einem Zeitintervall von 21 Tagen nicht beobachten. Ein dritter Versuch mit anderen Tumoren blieb ohne nennenswertes Ergebnis. 
Es ist also durchaus keine Konstanz der Erscheinungen zu beobachten. Manchmal scheint das Zeitintervall eine Rolle zu spielenl. Die wachstumsbefördernde Wirkung der Vorbehandlung mit artfremden Tumoren kommt scheinbar, wenn sie auftritt, immer bei Anwendung eines nur wenige Tage betragenden Zeitintervalles zwischen Vorbehandlung und Inplung zustande. Dagegen hat sich gerade bei der Vorbehandlung ron Vï̈usen mit Rattentumoren sowohl bei kürzerem als bei lïngerem Zeitintervall eine Immunitätswirkung gezeigt. Die Ursache fiir die Inkonstanz der Erscheinung liegt offensichtlich in biologischen Differenzen zwischen clen Tumoren, deren Kenntnis sich uns vorläufig noch entzieht. Wie die Immunität durch Vorimpfung mit artfremden Tumoren zustandekommt. lässt sich nicht olne weiteres sagen. Jedenfalls handelt es sich wohl doch um eine Antikiorperimmunität. In der Immunitätsforschung ist ja die Spezilitit der Antikörper im allgemeinen gewahrt. Doch kommen hier eine Reihe ron Ausnahmen vor. Schon bei der Gruber-Widalschen Typhusrealition zeigt sich manchmal, dass auch dem Typhuserreger mahestehende Bakterienarten mit dem Typhusserum agglutiniert werden. Ja es gibt sogar Fälle, wie sich besonders bei den Infektionen mit dem Gärtnersehen Enteritisbazillus gezeigt hat, wo sich das Serum eines Kranken dem eigentlichen Krankheitserreger gegenüber als weniger wirksam erweist, als gegenüber einer verwandten Bakterieuart. Bei den Präzipitinen liegen die Dinge ähnlich. Immunsera von Tieren, denen menschliches Blut oder Blutserum injiziert wurde, geben nicht nur mit Menschenblutserum sondern auch mit dem Serum von Affen und manchmal sogar mit weniger nahestehenden Tierarten ein typisches Präzipitin (P. Th. lïiller). Dasselbe kann man bei der Hämolyse beobachten.

Ein gegen Hammelblutkörperchen bei Ziegen hergestelltes Hämolysin löst manchmal auch rote Blutkörperchen ron Ziegen selbst auf. Es sind also die Antikörper nicht in dem Sinne streng artspezifisch, dass ein gegen bestimmte Bakterien oder gegen Zellen odẹr Eiweisssubstanzen einer Tierart wirksamer Antikörper nicht auch gegen andere Bakterien bzw. gegen Zellen oder Körperflüssigkeiten anderer Tierarten eine gewisse Wirksamkeit entfaltet. So kömnte man sich sehr wohl vorstellen. dass die durch Injektion der Premden Tumorzellen sich bildenden Antikörper anch gegen arteigene Tumorzellen wirksam sind, dass also Isocytolysine gebildet werden, so wie das Serum der gegen Hammelblntkörperchen vorbehindelten Ziegen manchmal auch Isohämolysine bildet. Es gibt ferner eine Reihe ron Beobachtungen in der Immunitätslehre, welche zeigen. dass es auch eine Organspezitizität der Antikörper gibt. die sich darin äussert, dass die Injektion einzelner Organe z. B. wie Uhlenhuth nachwies, der Kristalllinse des Auges, ferner der männlichen und weiblichen Geschlechtszellen, im 
Serum des Versuchstieres Antikörper erzeugt, welche gegen die entsprechenden Zellen der verschiedensten Tierarten wirksam sind, so dass also ein gegen die Kristalllinse des Menschen hergestelltes Immunserum auch mit den Linsenzellen der verschiedensten Tierarten reagiert. So wäre denn auch der Schluss berechtigt, dass bei der Bildung von Antikörpern gegen Tumorzellen eine gewisse Organspezifizität besteht, derart, dass nach der Injektion eines Tumors einer Tierart in manchen Fällen auch Immunitätsreaktionen mit den Tumorzellen anderer Tierarten stattfinden können. Es könnte sich auch bei den Immunitätsreaktionen nach der Vorbehandlung mit fremden Tumorzellen um einen von Paltauf als Zustandsspezifizität gekennzeichneten Vorgang handeln. Obermeyer und Pick fanden, dass Eiweisskörper, welche in irgend einer Weise chemisch veründert werden, sei es durch Erhitzung, Jodierung, Nitrierung oder andere Manipulationen, Antikörper bilden, welche gegen alle in gleicher Weise veründerte Körper, gleichgiltig von welcher Tierart sie herstammten, Antikörper zu bilden im Stande waren. Da nun die Tumorzellen in ihrer chemischen Konstitution sicher von den normalen Körperzellen differieren, könnte hier ein ähnlicher Vorgang sich abspielen, wenn man voraussetzt, dass die chemischen Differenzen aller Tumoren im Prinzip die gleichen sind. Die von Moreschi gefundene Tatsache, dass auch die Vorbehandlung mit artfremdem normalem Gewebe Immunitätsreaktionen bei der nachfolgenden Impfung mit dem arteigenen Tumor auslöst, spricht freilich mẹehr im Sinne der erst entwickelten Anschauung, dass durch die Injektion des fremden Tumors auch zugleich Isocytolysine gegen die Zellen des arteigenen Tumors gebildet werden. Die Ueberempfindlichkeitserscheinungen, welche Bashford, Moreschi und in manchen Fällen auch ich selbst nach der Vorbehandlung mit artfremden Tumorzellen beobachten konnten, erklären sich ganz analog den Vorgängen, welche wir bei der Immunisierung iuberhaupt kennen. Die anaphylaktischen Reaktionskörper sind, wie Paul Th. Müller ausführt, ja nichts anderes als Immunkörper besonderer Art, welche infolge der sensibilisierenden Antigeneinspritzung gebildet werden. Besonders durch Friedberger und Friedemann kennen wir neuerdings das Wesen der Anaphylaxie genauer.

Es geht aus ihren Versuchen hervor, dass die Bildung des anaphylaktischen Giftes durch Abspaltung aus der Antikörper-Antigen-Komplementverbindung erfolgt. Die anaphylaktischen Antikörper werden in den Organen gebildet, und es ist daher anzunehmen, dass eine wenige Tage nach der Vorbehandlung erfolgte Impfung zu einer Veründerung der Organzelle führt, welche eine Ueberempfindlichkeit im Sinne eines besseren Wachstums der Tumoren zur Folge hat.

Flexner und Jobling haben ja bei ihrem Rattentumor einen das 
Wachstum begünstigenden Einfluss ron Tumormaterial feststellen können, wenn sie es auf $5 f^{\circ}$ vorher erhitzt hatten und dann zur Vorimpfung verwendeten. Ich habe ebenfalls bei einem Rattentumor beobachten können, dass eine innerhalb weniger Tage rorgenommene Doppelimpfung einen erheblichen Wachstum befördernden Einfluss hatte. Aehnliches hat atteh v. Gierke gesehen. Hier haben wir also auch bei Verwendung artgleicher Tumoren anstatt einer nach der ersten Impfung zu erwartenden Immunität eine Ueberempfindlichkeit. Biologische Unterschiede zwischen den Tumoren erklären die Tatsache, dass das eine Mal Immunitätserscheinungen, also auch Ueberempfindlichkeitserscheinungen eintreten, das andere Mal nicht. Die Ueberempfindlichkeit äussert sich bei den Tumoren in einer Steigerung ihrer Virulenz. Ich habe aber im Verlaufe dieser Arbeiten auch Erscheinungen konstatieren können, welche im Simne der in der Serologie nach den verschiedensten Immunisierungsversuchen beobachteten anaphylaktischen Realtionen (Giftwirkung) zu deuten sind.

Es zeigte sich nämlich sehr häufig bei der Injektion von artfremden Tumoren eine tötliche Wirkung der Injektion, die schon im Verlaufe von 21 Stunden zum Tode fast aller Versuchstiere führte. Die in solchem Falle überlebenden Tiere vertragen eine nach 4-6 Tagen vorgenommene zweite Injektion mit demselben Tumor meistenteils nicht mehr und gingen dann sämtlich ein, während derselbe Tumor bei der eigenen Tierart keine Wirkung zeigte. In sehr vịelen Fällen wurde diese zweite Injektion aber auch dann nicht vertragen, wenn nicht ein artfremder, sondern ein arteigener Tumor verwendet wurde. So konnte ich besonders bei Mäusen, welche die erste Injektion des Rattentumors gut rertragen hatten, eine in 24- 48 Stunden zum Tode führende Wirkung einer nach wenigen Tagen vorgenommenen Impfung mit Mäusecarcinom konstatieren, während die Impfung dieses selben Mänsecarcinoms bei den Kontrollmäusen irgend eine giftige Wirkung nicht hatte. Eine Erklärung für die Giftigkeit der ersten Injektion z. B. von Rattensarkom bei Müusen scheinen mir Angaben von Daels zu geben. Daels fand nämlich, dass nekrotisches Gewebe von Rattensarkomen stark toxische Eigenselialten hat, und zwar sowohl für refraktäre wie für emplängliche Tiere.

Wenn wir uns nun erinnern, dass nach Biedl und Kraus alle Erscheinungen der Anaphylaxie durch die einmalige Injektion von WittePepton hervorgerufen werden können, so wäre eine Erklärung der schon nach einer einmaligen Injektion von Tumorgewebe erfolgten tötlichen Wirkung insofern gegeben, als man annehmen kann, dass wir Substanzen injiziert haben, welche peptonähnlichen Charakter haben. Das wäre ja bei den autolytischen Vorgängen, die im Spiele sind. verständlich. Ist die Bildung dieses dem anaphylaktischen Gift, dem Anaphylatoxin. ähnlichen 
peptonartigen Körper in mehr oder minderem Grade weit erfolgt, so kommt es zur anaphylaktischen Giftwirkung schon nach der ersten oder erst nach der zweiten Injektion. Wenn die Giftwirkung auch dann eintritt, wenn wir zur ersten Injektion einen artfremden, zur zweiten jedoch den arteigenen Tumor verwenden, so gilt hier alles das, was wir bereits vorher ausgeführt haben. Auch hier wäre zu folgern, dass die Vorhehandlung mit artfremdem Tumorgewebe Immunitätsreaktionen gegen arteigene Tumoren auslösen kann. Denn auch die Anaphylaxiereaktionen sind ja Immunitätserscheinungen. 